\title{
MicroRNA and hyperaggregability of platelets in women with sticky platelet syndrome and pregnancy complications
}

\author{
Vadelova $\mathrm{L}^{1}$, Skerenova $\mathrm{M}^{2}$, Ivankova $\mathrm{J}^{1}$, Vazanova $\mathrm{A}^{1}$, Sokol $\mathrm{J}^{1}$, Zolkova $\mathrm{J}^{1}$, Stanciakova $\mathrm{L}^{1}$, \\ Skornova $\mathrm{I}^{1}$, Stasko $\mathrm{J}^{1}$ \\ Department of Hematology and Transfusiology, National Center of Hemostasis and Thrombosis, Jessenius Faculty of Medicine \\ in Martin, Comenius University in Bratislava, Martin University Hospital, Martin, Slovakia. vadelova2@uniba.sk
}

\begin{abstract}
AIMS: We aimed to characterize relationship between the expression profiles of platelet miR-96, miR-126 and miR-223 and platelet function examination in patients with sticky platelet syndrome (SPS) and in healthy controls.

BACKGROUND: MicroRNAs (miRNA, miR) are a group of small and non-coding RNAs involved in many mechanisms as regulators of post-transcriptional protein expression in platelets. SPS is defined as platelet hyperaggregability after administration of low doses of adenosine diphosphate and/or epinephrine. Clear genetic abnormality of this syndrome is not known yet.

METHODS: We examined 45 patients with SPS and 30 healthy volunteers. For functional platelet examination we used light transmission aggregometry, and qRT-PCR was used to determine the expression of the miRNAs.

RESULTS: We observed no relationship of the platelet miRNA expression with functional platelet examination in the entire cohort of patients with SPS. However, in a group of patients with SPS and pregnancy complications, we found that the expression of platelet miR-96 $(p=0.009)$ was up-regulated. CONCLUSION: In spite of the multiple limitations of the study, it can be considered that the increased expression of platelet miR-96 found in a group of patients with SPS and pregnancy complications could be related to the hyperaggregability in these selected patients (Tab. 2, Ref. 31). Text in PDF www.elis.sk KEY WORDS: sticky platelet syndrome, platelet miRNA, platelet hyperaggregability, magnetic platelet separation.
\end{abstract}

\section{Introduction}

\section{Sticky platelet syndrome}

The abnormal high platelet in vitro aggregation response to a low concentration of epinephrine (EPI) and/or adenosine diphosphate (ADP) is characterized as the sticky platelet syndrome (SPS). Effects of other inducers on platelet aggregation (arachidonic acid, collagen, thrombin and ristocetin) remain within normal range $(1,2)$. According to Mammen and Bick, there are

\footnotetext{
${ }^{1}$ Department of Hematology and Transfusiology, National Center of Hemostasis and Thrombosis, Jessenius Faculty of Medicine in Martin, Comenius University in Bratislava, Martin University Hospital, Martin, Slovakia, and ${ }^{2}$ Divison of Molecular Medicine, Biomedical Center Martin JFM CU, Jessenius Faculty of Medicine in Martin, Comenius University in Bratislava, Martin University Hospital, Martin, Slovakia

Address for correspondence: L. Vadelova, Department of Hematology and Transfusiology, National Center of Hemostasis and Thrombosis, Comenius University in Bratislava, Jessenius Faculty of Medicine in Martin, Martin University Hospital, Kollarova 2, SK-036 01 Martin, Slovakia. Phone: +421.4203696

Acknowledgement: This study was supported by projects APVV 16-0020, APVV 17-0054, VEGA 1/0187/17, VEGA 1/0549/19 and UC GRANT 1916/2016.mmm
}

3 types of SPS: the in vitro platelet hyperaggregation after ADP and/or EPI is classified as type I, hyperaggregation after EPI alone is classified as type II, and hyperaggregation after ADP alone defines type III. SPS type II seems to be the most common form (3, $4,5)$. In the development of angina pectoris, acute myocardial infarction (MI), transient cerebral ischemic attacks, stroke, ischemic optic neuropathy, and venous thrombosis, the SPS has been established as a factor playing a role, however not all carriers of the syndrome experience clinical symptoms (6). SPS is generally described as an inherited platelet disorder with an autosomal trait. However, not all patients have a positive family history (2). At the same time, the aggregation pattern and laboratory type of the syndrome can vary among the affected members of the same family (7), and the genetic studies have not identified a specific genetic mutation universally presented in all patients with this syndrome. Moreover, various mutations of the same or various genes resulting in a similar phenotype could be associated with certain acquired conditions (8). This can support the idea about multifactorial etiology of SPS. In regard to SPS and pregnancy complications, Yagmur et al have shown for the first time in a major recent retrospective study that SPS is a frequent disorder in young women with infertility and unexplained pregnancy losses in their history. This study proves a high prevalence of SPS type 
Tab. 1. Overview of platelet microRNA and their function within platelets.

\begin{tabular}{lllll}
\hline MicroRNA & Target & Role in platelets & Study samples & Study \\
\hline miRNA-223 & $\begin{array}{l}\text { Factor XIII } \\
\text { P2Y }_{12} \text { receptor }\end{array}$ & $\begin{array}{l}\text { Platelet } \\
\text { aggregation }\end{array}$ & $\begin{array}{l}\text { Mice, } \\
\text { Human platelets }\end{array}$ & $(24,25)$ \\
\hline miRNA-126 & $\begin{array}{l}\text { Disintegrin and metalloproteinase } \\
\text { domain-containing protein 9, } \\
\text { Plexin-B2 }\end{array}$ & $\begin{array}{l}\text { Platelet } \\
\text { reactivity }\end{array}$ & $\begin{array}{l}\text { Human } \\
\text { megakaryocytes }\end{array}$ & $(19,27)$ \\
& VAMP8 (endobrevin) & $\begin{array}{l}\text { Platelet } \\
\text { reactivity }\end{array}$ & Human platelets & $(20)$ \\
\hline miRNA-96 & & & \\
\hline
\end{tabular}

miRNA - microRNA; VAMP8 - vesicle-associated membrane protein 8; P2 ${ }_{12}$ - ADP receptor

has a role in the heterogeneity of platelet reactivity, and suggested that miR-96 has a role in the regulation of the VAMP8 expression at protein and RNA levels (20) (Tab. 1). Their finding that VAMP8 expression is associated with increased platelet reactivity in humans is in accordance with the earlier published work of Polgar et al which shows a role for VAMP8 in granule secretion (21).

Due to the above findings on platelet

II (33.2 \%) in 208 affected young females with pregnancy complications. Moreover, the group of women with SPS treated with a low dose of acetylsalicylic acid (ASA) showed an improved pregnancy outcome compared to women with SPS and without the low-dose ASA therapy $(9,10)$.

In our study, we would like to verify if some changes in the platelet microRNA expression can be associated with SPS.

\section{Platelet miRNA}

In 1993, Lee et al accidentally discovered small endogenous ribonucleotide acids (RNAs), micro ribonucleotide acid (miRNA, $\mathrm{miR}$ ), in the research of the timing of stages of Caenorhabditis elegans nematodes. The role of these small miRNAs consisting of $\sim 22$ nucleotides lies in transcriptional regulation of gene expression by pairing to the 3'untranslated region (3'UTR) (11). Because of the regulation exerted by miRNAs in numerous biological processes such as proliferation, apoptosis and differentiation, the impairment of the MiRNA regulatory network may be involved in various disorders including tumorigenesis, vascular and platelet disorders, etc. $(12-14)$. Platelets have critical roles in normal hemostatic processes and pathologic conditions as thrombosis, vascular remodeling, inflammation and wound repair (15). Today, more and more studies are interested in platelet miRNA. Platelets are an abundant source of miRNA (16), and platelet reactivity correlates with platelet miRNA expression profiles (Tab. 1) (17). By microarray screening, miR-126, miR-223, miR-197, miR-24 and miR-21 were discovered as the most highly expressed miRNAs in platelets and platelet microparticles (18). The ability of miR-223 to bind to the 3'UTR of human P2Y ${ }_{12}$ receptor mRNA is very important because the platelet functions that are as critical as aggregation and granule secretion activated by ADP, are mediated by the $\mathrm{P} 2 \mathrm{Y}_{12}$ receptor. The results from Landry et al are suggesting that miR-223 could regulate $\mathrm{P} 2 \mathrm{Y}_{12}$ receptor levels because of coprecipitation of platelet $\mathrm{P} 2 \mathrm{Y}_{12}$ receptor mRNA with the Argonaute2 (Ago2) protein. According to this study, more $\mathrm{P}_{2} \mathrm{Y}_{12}$ receptor activities caused by lower levels of platelet miR-223 will appear as a higher platelet activity (16).

As mentioned earlier, miR-126 was also established as the most highly expressed miRNA in platelets. According to study results of Kaudewitz et al, platelet aggregation in mice is reduced by inhibition of miR-126, and the expression of ADAM9 and $\mathrm{P} 2 \mathrm{Y}_{12}$ receptors is directly and indirectly affected by miR-126 (19).

Kondkar et al showed that protein VAMP8/ endobrevin, an important protein which is involved in platelet degranulation,
miRNA, we chose miR-223, miR-126 and miR-96 to verify their expression in patients with SPS.

\section{Material and methods}

\section{Ethics statement and subjects}

Blood collection from patients and healthy volunteers was approved by decision of the Ethics Committee of JLF UK according to EK 1916/2016. All study subjects agreed to participate in this project and confirmed their agreement by signing informed consent in accordance with the Helsinki Declaration.

This study included 45 patients with SPS (type I, type II) and 30 healthy volunteers. The diagnosis of SPS was confirmed or disproved by light transmission aggregometry (LTA) in all subjects, while all of them had initial platelet counts within the reference range. Aggregometry testing was performed in all subjects without antiplatelet therapy. The administration of ADP inhibitor or ASA treatment was interrupted for more than 7 days before testing, and the use of other drugs with a possible effect on platelet activity was omitted with a negative diagnosis of SPS.

\section{Diagnosis of SPS by Mammen}

The peripheral venous blood was collected into the tubes with $3.2 \%$ sodium citrate to assess the platelet aggregation. The samples were processed and analyzed within 2 hours after blood samples collection according to Mammen as described previously (4). The platelet function was evaluated by testing the aggregation responses to 3 low concentrations of $\operatorname{ADP}(2.34,1.17$, and 0.58 $\mathrm{mmol} / \mathrm{L})$ and $\mathrm{EPI}(11.0,1.1$, and $0.55 \mathrm{mmol} / \mathrm{L})$ with platelet-rich plasma (PRP) and platelet-poor plasma (PPP) using LTA (AggRAM, Helena Laboratories, USA).

\section{Leukocyte-depleted and erythrocyte-depleted platelet prepara-} tion

Leukocyte and erythrocyte depleted platelets were prepared from the peripheral venous blood collected into the tubes with $3.2 \%$ sodium citrate. Before centrifugation and handling the samples, the prostaglandin E1 was added into these samples to prevent platelet activation. By centrifugation at $200 \mathrm{x}$ g for 10 minutes at $37^{\circ} \mathrm{C}$, PRP was obtained, only the upper $9 / 10$ of PRP volume was used to minimize leukocyte and erythrocyte contamination. Platelets were pelleted at $400 \mathrm{x} g$ for 10 minutes at $37^{\circ} \mathrm{C}$ and subsequently gently resuspended in TRIS buffer. CD45 MicroBeads and CD235a MicroBeads reagents were added and 


\section{0-704}

incubated at room temperature for 15 minutes in the dark. Magnetic separation columns were used to entrap CD45 (leukocyte fraction) and $\mathrm{CD} 235 \mathrm{a}^{+}$(erythrocyte fraction) by positive selection (MidiMACSTM Separator, MiltenyiBiotec, Zürich, Switzerland). RNA stabilization solution RNAlater ${ }^{\circledR}$ Tissue Collection (Life Technologies Corporation, Carlsbad, USA) was added into purified platelets and concentrated by centrifugation at $400 \mathrm{x}$ g for 10 minutes at $37^{\circ} \mathrm{C}$. The samples were stored at $-80^{\circ} \mathrm{C}$.

\section{Platelet miRNA analysis}

The isolation of miRNA from pure platelets was performed with High Pure miRNA Isolation Kit (F. Hoffmann-La Roche Ltd, Basel, Switzerland) according to manufacturer's instructions modified for work with platelets. The isolation step was controlled using RNA Spike-in (UniSP2, UniSP4 and UniSP5 RNA) (QIAGEN GmbH, Hilden, Germany) and reverse transcription using UniSP6 Spike-in. The reverse transcription was performed using miRCURY LNA RT Kit (QIAGEN GmbH, Hilden, Germany) in duplexes. Targeted miRNAs (hsa-miR-96-5p, hsa-miR-126-3p and hsa-miR-223-3p), endogenous control (hsa-miR-18a-5p, hsamiR-148b-3p) and Spike-in miRNAAssays were performed using miRCURY LNA miRNA PCR Assay (QIAGEN GmbH, Hilden, Germany). For normalization, endogenous control was selected after analysis of candidate reference miRNAs. We chose the most optimal combination to evaluate the expression of analyzed miRNAs, namely the combination of hsa-miR-18a-5p together with hsa-miR-148b-3p.

\section{Statistical analysis}

Basic descriptive statistics and statistical tests were performed in Microsoft Excel spreadsheet (2019). The data obtained from qRT-PCR were processed in the QIAGEN GeneGlobe Data analysis center (http://geneglobe.qiagen.com/en/analyze/). Results with $\mathrm{p}<0.05$ were considered statistically significant.

\section{Results}

Using qRT-PCR, we determined the expression of hsa-miR126-3p, hsa-miR-223-3p and hsa-miR-96-5p, whose values were normalized by the combined endogenous control of hsa-miR$18 \mathrm{a}-5 \mathrm{p}$ with hsa-miR-148b-3p. Expression results of each are expressed as mean $\mathrm{Ct}$ value (cycle threshold). The differences in relative expression levels of the targeted miRNAs between SPS patients and controls were tested and relative fold changes for each miRNA were determined. The results of comparison for the whole study population of SPS patients were then divided into groups according to the type of SPS (type I and type II). The results of comparison between SPS women associated with pregnancy complications and healthy controls are shown in Table 2. There were no statistically significant changes in miRNA's expression levels between groups. The only exception was the group of SPS women associated with pregnancy complications. As in previous tests, the expression levels of hsa-miR-126-3p and hsa-miR-223-3p in this female group were not different from those in the healthy control group. However, the hsa-miR-96-5p expression levels in SPS women with a recurrent miscarriage were significantly increased $(p=0.009)$. The expression of hsa-miR-96-5p in patients with SPS and pregnancy complications was almost 2 -fold higher than that in the healthy control group.

\section{Discussion}

SPS is a common cause of otherwise unexplained thrombosis and pregnancy complications. The cause of this syndrome remains unknown although it is defined by precise clinical and laboratory criteria. However, even the currently published studies have failed to identify a common genetic defect for all individuals with SPS. Nevertheless, the results of Sokol et al suggest that the platelet hyperaggregability in patients with SPS and history of miscarriage may be associated with the variability of the GP6, GAS6 and PEAR1 genes (1).

The content of non-coding RNA could be an important parameter reflecting the reactivity of the intrinsic properties of platelets. It is known that miRNA expression values in healthy humans do not change during platelet life but miRNA expression levels differed in patients suffering from various diseases such as diabetes mellitus, cancer and MI $(22-25,12-14)$. In recent years, the importance of non-coding RNA molecules has come to the fore. Most studies have focused on miRNAs, but paradoxically, there is very little information about the role of these molecules in the biology of megakaryocytes and platelets. Studies on miR-96, miR-126, and miR-223 show the association of miRNA and platelet function (14, 19). In this work we focused on the potential role of the abovementioned platelet miRNAs as a biomarker of platelet hyperaggregability, and as a possible player in the pathogenesis of SPS.

The main aim of our study was to determine if there can be a relationship between functional platelet examination (platelet aggregation after induction with low concentrations of ADP and /or EPI) and results of platelet miR-96, miR-126 and miR-233 analysis in SPS patients and healthy controls. The results of these examinations in the entire cohort of SPS patients and healthy controls were compared as well as those in patients with SPS and pregnancy complications vs. healthy controls.

Tab. 2. Results of statistical analysis of platelet miRNA expression levels differences between various groups and healthy controls.

\begin{tabular}{|c|c|c|c|c|c|c|c|c|}
\hline \multirow{2}{*}{ miRNA ID } & \multicolumn{2}{|c|}{ Whole study subjects } & \multicolumn{2}{|c|}{ SPS type I } & \multicolumn{2}{|c|}{ SPS type II } & \multicolumn{2}{|c|}{ SPS women } \\
\hline & Fold change & $\mathrm{p}$ & Fold change & $\mathrm{p}$ & Fold change & $\mathrm{p}$ & Fold change & $\mathrm{p}$ \\
\hline hsa-miR-96-5p & 0.77 & 0.690 & 1.10 & 0.111 & 0.68 & 0.225 & 1.85 & 0.009 \\
\hline hsa-miR-126-3p & 1.13 & 0.842 & 1.00 & 0.564 & 1.12 & 0.496 & 0.98 & 0.646 \\
\hline hsa-miR-223-3p & 1.13 & 0.931 & 0.99 & 0.460 & 1.14 & 0.493 & 0.98 & 0.628 \\
\hline
\end{tabular}

miRNA - microRNA; hsa-miR - homo sapiens miRNA; SPS women - SPS young women with pregnancy complications 


\section{MiR-126}

Although miR-126 is enriched in endothelial cells and promotes endothelial integrity, its circulating values cannot be attributed only to endothelial cells. In fact, miR-126 is also expressed in megakaryocytes, while miR-126 circulating values appear to be predominantly derived from platelets (26-27). The association between miR-126 and platelet responses has been discussed by Kaudewitz et al. In his study, he investigated the role of miR-126 in vivo in mouse platelets. Following treatment with the miR-126 inhibitor, the aggregation response upon induction by arachidonic acid and U9887 was decreased (19). In our study, we also focused on miR-126 because of its abundance in platelets and its ability to regulate platelet reactivity. When compared to healthy controls, the SPS patients showed no significant change in platelet miR-126 expression. Also, platelet miR-126 expression changed neither in the subgroups of SPS types, nor in SPS patients with pregnancy complications.

\section{$\operatorname{MiR}-223$}

MiR-223 targets the 3'UTR region of the human platelet mRNA receptor $\mathrm{P} 2 \mathrm{Y}_{12}$. The $\mathrm{P} 2 \mathrm{Y}_{12}$ receptor is important in platelet activation and it is the target of many drugs used to inhibit platelet function. Lower expression of platelet miR-223 may lead to greater $\mathrm{P} 2 \mathrm{Y}_{12}$ receptor activity and thus to higher platelet reactivity. In this way, decreased levels of platelet miR-223 expression may increase the risk of thrombotic disease such as MI (14). This may also support the observation that lower expression of platelet miR-223 is associated with decreased efficacy of $\mathrm{P}_{2} \mathrm{Y}_{12}$ receptor antagonists such as clopidogrel. Duan et al reported that the expression of platelet miR-223 and miR-146a was lower in stroke patients suffering from concomitant diabetes mellitus as compared to controls. The platelet miR-223 and miR-146a expression significantly correlated with platelet reactivity as measured by P-selectin expression, and also correlated with P-selectin plasma levels, suggesting that platelets are a major source of circulating miR-223 (23). Landry et al have shown that platelet receptor P2 $\mathrm{Y}_{12}$ mRNA co-precipitates with Ago2, suggesting that miR-223 could regulate the receptor levels and thus also the platelet function (12). In our study, we selected to analyse miR-223 for its ability to regulate the ADP receptor. However, according to our results, there was no statistically significant change in the expression of miR-223 values in any of the SPS groups, so we assume that there is no significant relationship between platelet miR-223 expression values and platelet hyperreactivity in SPS.

\section{MiR-96}

In 2010, Kondkar et al reported data about the role of miR-96 as a regulator of platelet activity. According to their results, miR-96 can regulate the membrane protein endobrevine (VAMP8), an important protein that plays a part in the platelet degranulation reaction. MiR-96 expression values were 2.6-fold higher in hyporeactive platelets (20).

However, there was a considerable inconsistency and heterogeneity concerning the classification of Kondkar study patients according to the hyperreactive and hyporeactive platelet group as compared to our study group. Kondkar et al used a different con- centration of epinephrine $(1.5 \mathrm{mM})$ for LTA to distinguish hyperreactive platelets than was used in our study $(0.55 \mathrm{mM}, 1.1 \mathrm{mM}$ and $11.0 \mathrm{mM}$ ). The authors in this study determined the cut-off level for hyperreactive vs. hyporeactive platelets using LTA as a platelet response after induction with $1.5 \mathrm{mM}$ epinephrine $>60 \%$ vs $<40 \%$ of aggregation, while we determined the cut-off level for hyperreactive platelets $>30 \%$ of platelet aggregation using LTA and $1.1 \mathrm{mM}$ epinephrine. From our point of view, there were at least some patients in Kondkar study classified as hyporeactive that could have been evaluated as normo/hyperreactive according to Mammen criteria $(4,28)$ (used in our study).

In contrast, Shi et al monitored the platelet activity index in patients with acute coronary syndrome without ST segment elevation treated with aspirin and clopidogrel and reported different results of platelet miR-96 expression. This study found unchanged miR-96 expression values in patients who had a decreased response to clopidogrel and demonstrated no association between platelet response to clopidogrel and miR-96 expression. The study also points out that miR-96 is not one of the miRNAs with highest expression in human platelets. Nevertheless, the authors have not discredited the regulatory role of miR-96 in platelet reactivity, although this information needs to be validated and verified by a larger study (29).

In our study, the platelet miR-96 expression did not differ significantly between the group of all patients with SPS (without division into SPS subtypes) and healthy controls. The platelet miR-96 expression reached statistical significance in neither of the subgroups of SPS (SPS I and SPS II) as compared to healthy controls. Interestingly though, in the group of patients with SPS (without splitting to SPS types) and pregnancy complications, there was a significantly increased platelet miR-96 expression in comparison with controls $(\mathrm{p}<0.01)$ (Tab. 2). Increased platelet miR-96 expression could have affected platelet aggregation in patients with SPS and thus could have contributed to the development of pregnancy complications. However, there are many risk factors that can lead to repeated fetal loss, such as anatomical, hormonal, immunological, or chromosomal abnormalities (30). Among the fetal loss risk factors, the thrombophilic conditions deserve special attention. Low blood pressure and turbulent blood flow in the placenta, along with changes in hypercoagulation ability during this period, may predispose women to thrombosis. It is possible that some hypercoagulation conditions may lead to arterial thrombosis, which may result in uteroplacental thrombosis and obstetric complications. However, the exact mechanism is unknown (31). Thus, in our study, the expression of platelet miR-96 in young SPS women with recurrent fetal losses was found to be almost twice as high as that found in controls. The increased platelet miR-96 in the hyperreactive platelets of young women with SPS and pregnancy complications seems to be challenging because contrariwise, Kondkar et al (2010) reported that the platelet miR-96 expression was increased in hyporeactive platelets in healthy population of blood donors. This discrepancy can be explained by the heterogeneity of studied groups, study design, methods and study protocol used. One of the reasons why no difference was observed in the platelet expression of miR-96 may be that the LTA and even VASP analysis are not specific for miR-96-regulated pathways (29). 
700-704

In particular, the limitation of our study is a low number of enrolled patients with SPS. We assume that this limitation is caused partially by the fact that SPS is relatively rare in the Slovak population, but also due to the complicated sample collection associated with the need to enroll patients before the start of treatment. Another limitation of the study could lie in inconsistencies in the genetic analysis methodology, platelet separation methodology, as well as overall work with platelets.

In spite of the multiple limitations of the study, it can be proposed that the increased expression of platelet miR-96 found in the group of patients with SPS and pregnancy complications could be related to hyperaggregability in these selected young women. Further research in this area is awaited.

\section{References}

1. Sokol J, Skerenova M, Jedinakova $Z$ et al. Progress in the understanding of sticky platelet syndrome. Semin Thromb and Hemost 2016; 43 (1): 8-13.

2. Kubisz P, Stasko J, Holly P. Sticky platelet syndrome. Semin Thromb Hemost 2013; 39 (6): 674-683.

3. Bick RL. Sticky platelet syndrome: A common cause of unexplained arterial and venous thrombosis. Clin Appl Thromb Hemost 1998; 4: 77-81.

4. Mammen EF. Ten years' experience with the "Sticky platelet syndrome". Clin Appl Thromb Hemost 1995; 1: 66-72.

5. Holiday PL, Mammen E, Gilroy J. Sticky platelet syndrome and cerebral infarction in young adults. The Ninth International Joint Conference on Stroke and Cerebral Circulation 1983, Phoenix: 1983 (abstr.). In: Bick RL (Editor). Disorders of Thrombosis and Hemostasis. Clinical and Laboratory Practice. 3rd Ed., Lippincott, Williams \& Wilkins, Philadelphia 2002, 453 p.

6. Škereňová M, Sokol J, Biringer J et al. GP6 Haplotype of missense variants is associated with sticky platelet syndrome. Clin Appl Thromb Hemost 2018; 24 (1): 63-69.

7. Šimonová R, Bartosová L, Chudý P et al. Nine kindreds of familial sticky platelet syndrome phenotype. Clin Appl Thromb Hemost 2013;19 (04): 395-340.

8. Kubisz P, Holly P, Stasko J. Sticky Platelet Syndrome: 35 Years of Growing Evidence. Semin Thromb Hemost 2019 ; 45 (1): 61-68.

9. Yagmur E, Bast E, Mühlfeld AS et al. High Prevalence of Sticky Platelet Syndrome in Patients with Infertility and Pregnancy Loss. J Clin Med 2019; 8 (9): 1328. DOI:10.3390/jcm8091328.

10. Sokol J, Kubisz P, Stasko J. Comment on: Inherited Thrombophilia and Pregnancy Complications: Should We Test? Semin Thromb Hemost 2020; Epub ahead of print, accepted 26.12.2019. DOI: 10.1055/ s-0039-3402479.

11. Lagos-Quintana M, Rauhut $R$, Lendeckel $W$ et al. Identification of novel genes coding for small expressed RNAs. Science 2001; 294 (5543): 853-858.

12. Taherdangkoo K, Kazemi Nezhad SR, Hajjari MR et al. miR-485$3 p$ suppresses colorectal cancer via targeting TPX2. Bratisl Med J 2020; 121 (4): 302-307.

13. Yurt M, Ayyildiz O, Karakus A et al. MicroRNAs expression profiles as biomarkers and therapeutic tools in Turkish patients with chronic myeloid leukemia. Bratisl Med J 2020; 121 (2): 159-163.
14. Nishiguchi T, Imanishi T, Akasaka T. MicroRNAs and cardiovascular diseases. BioMed Res Int 2015; Article ID 682857, 14 p. doi. org/10.1155/2015/682857.

15. Gnatenko DV, Dunn JJ, McCorkle SR et al. Transcript profiling of human platelets using microarray and serial analysis of gene expression. Blood 2003; 101 (6): 2285-2293.

16. Landry P, Plante I, Ouellet DL et al. Existence of a microRNA pathway in anucleate platelets. Nat Struct Mol Biol 2009; 16: 961-966.

17. Nagalla $S$, Shaw $C$, Kong $X$ et al. Platelet microRNA-mRNA coexpression profiles corre-late with platelet reactivity. Blood 2011; 117: 5189-5197.

18. Paseban M, Marjaneh RM, Banach M et al. Modulation of microRNAs by aspirin in cardiovascular disease. Trends Cardiovasc Med 2019; Epub ahead of print, accepted 14.8.2019. DOI: 10.1016/j.tcm.2019.08.005.

19. Kaudewitz D, Skroblin P, Bender LH et al. Association of MicroRNAs and YRNAs with Platelet Function. Circ Res 2016; 118: 420-432.

20. Kondkar AA, Bray MS, Leal SM et al. VAMP8/endobrevin is overexpressed in hyperreactive human platelets: suggested role for platelet microRNA. J Thromb Haemost 2010; 8: 369-378.

21. Polgar J, Chung SH, Reed GL. Vesicle-associated-membrane protein 3 (VAMP-3) and VAMP-8 are present in human platelets and are required for granule secretion. Blood 2002; 100: 1081-1083.

22. Deng J, Guo F. MicroRNAs and type 2 diabetes. ExRNA 2019; 1: 36. doi.org/10.1186/s41544-019-0038-5

23. Duan X, Zhan Q, Song B et al. Detection of platelet microRNA expression in patients with diabetes mellitus with or without ischemic stroke. J Diabetes Complications 2014; 28: 705-710.

24. Elgheznawy A, Shi L, Hu J et al. Dicer cleavage by calpain determines platelet microRNA levels and function in diabetes. Circ Res 2015; 117: $157-165$.

25. Fejes Z, Póliska $\mathbf{S}$, Czimmerer $\mathbf{Z}$ et al. Hyperglycaemia suppresses microRNA expression in platelets to increase P2RY12 and SELP levels in type 2 diabetes mellitus. Thromb Haemost 2017; 117 (03): 529-542.

26. Sunderland N, Skroblin P, Barwari T et al. MicroRNA biomarkers and platelet reactivity: the clot thickens. Circ Res, 2017; 120: 418-435.

27. Garcia A, Dunoyer-Geindre S, Zapilko V et al. Functional validation of microRNA-126-3p as a platelet reactivity regulator using human haematopoietic stem cells. Thromb Haemost, 2019; 119 (2): 254-263.

28. Mammen EF, Barnhart MI, Selik NR et al. "Sticky platelet syndrome": a congenital platelet abnormality predisposing to thrombosis? Folia Haematol Int Mag Klin Morphol Blutforsch 1988; 115 (3): 361-365.

29. Shi R, Ge L, Zhou $X$ et al. Decreased platelet miR-223 expression is associated with high on-clopidogrel platelet reactivity. Thromb Res 2013; 131: 508-513.

30. Sokol J, Biringer K, Skerenova $M$ et al. Platelet aggregation abnormalities in patients with fetal losses: the GP6 gene polymorphism. Fertil Steril, 2012; 98 (5): 1170-1174.

31. Sokol J, Skerenova M, Biringer K et al. Glycoprotein VI Gene Variants Affect Pregnancy Loss in Patients With Platelet Hyperaggregability. Clin Appl Thromb Hemost 2018; 24 (9 Suppl): 202S-208S.

Received May 11, 2020. Accepted June 10, 2020. 